\title{
Thermophoretic force on micro- and nanoparticles in dilute binary gas mixtures
}

\author{
Jun Wang and Zhigang Li \\ Department of Mechanical Engineering, The Hong Kong University of Science and Technology, Clear Water Bay, Kowloon, Hong Kong
}

(Received 27 April 2011; published 3 August 2011; corrected 3 August 2011)

\begin{abstract}
We theoretically investigate the thermophoretic force on spherical particles in binary monatomic gas mixtures in the free molecular regime. Based on gas kinetic theory and by considering the gas-particle interactions, we derive the analytical formulas of the thermophoretic force for two limiting gas-particle collision models; namely, specular and diffuse scattering scenarios. The formulas are consistent with those in simple gases and apply to both micro- and nanoparticles; for the latter the intermolecular interactions are important. As an example, the thermophoretic force on $\mathrm{Ag}$ nanoparticles in He-Ar mixtures is illustrated.
\end{abstract}

DOI: 10.1103/PhysRevE.84.021201

PACS number(s): 51.10.+y, 47.45.Dt, 05.60.Cd, 65.80.-g

\section{INTRODUCTION}

Thermophoresis is a phenomenon where small particles suspended in a fluid (usually a gas) with a nonuniform temperature distribution tend to move from high to low temperature. It is an old scientific problem going back to around the mid 19th century $[1,2]$. The force that drives the particles to move against the temperature gradient is called the thermophoretic force. In steady state, the particles move with a constant velocity (thermophoretic velocity) when the thermophoretic force is balanced by the drag force, which is due to the particle-fluid relative motion and the viscosity gradient in the fluid $[3,4]$. Thermophoresis plays important roles in many areas, including aerosol science, biology, and the semiconductor industry (see Ref. [5] and references cited therein). Most of the applications employ the thermophoretic force to manipulate or separate suspended objects, such as microparticles and biomolecules. This positions the thermophoretic force as a key issue in practical applications.

In gaseous media, the thermophoretic force acting on a suspended object depends on the flow regimes, which are characterized by the Knudsen number $\mathrm{Kn}=\lambda / L$, where $\lambda$ is the mean-free path of the gas and $L$ is the characteristic length of the object. In the past decades, extensive theoretical, experimental, and numerical investigations have been conducted to understand the phenomenon of thermophoresis [5-31]. For theoretical analysis, the calculation of the thermophoretic force usually involves solving the Boltzmann equations. This is especially difficult in the continuum $(\mathrm{Kn} \ll 1)$ and transition $(\mathrm{Kn} \sim 1)$ regimes, where the velocity distribution of the gas molecules is greatly affected by the motion of the objects and the theoretical analysis is very complex. Although a few analytical approaches have been developed based on different approximation methods [5,6,9], the theories in these two regimes are far from complete. In the free molecular regime $(\mathrm{Kn} \gg 1)$, the problem can be greatly simplified by assuming that the presence of the suspended objects does not affect the velocity distribution of the gas molecules and that pair collisions of gas molecules dominate. In this case, the Chapman-Enskog gas-kinetic theory [32-34] for dilute gases in a nonequilibrium state can be used to obtain the thermophoretic force.

In the free molecular regime, the thermophoretic force on spherical particles in simple monatomic gases under the assumption of rigid body collisions (i.e., gas molecules do not interact with the particles unless they are in physical contact), was developed by Waldmann [10],

$$
\mathbf{F}_{T}=-\frac{8}{15} \sqrt{\frac{2 \pi m_{g}}{k_{B} T}} \kappa R^{2} \nabla T,
$$

where $m_{g}$ is the mass of the gas molecule, $k_{B}$ is the Boltzmann constant, $\kappa$ is the thermal conductivity of the gas, $R$ is the particle radius, and $T$ is the temperature. For microparticles, Eq. (1) agrees well with experiments and numerical simulations [16-19]. For nanoparticles, however, the rigid body collision assumed by Waldmann becomes questionable due to the fact that the interaction between gas molecules and particles could be important [3,26,27]. By considering the intermolecular interactions, $\mathrm{Li}$ and Wang derived the thermophoretic force in simple gases based on a rigorous gas-kinetic analysis [27]. For specular and diffuse scatterings of gas molecules upon collisions with a nanoparticle, the thermophoretic forces are given by

$$
\mathbf{F}_{T, s / d}=\frac{8}{3} \sqrt{\frac{2 \pi m_{r}}{k_{B} T}} \kappa R^{2} \nabla T\left(\Omega_{s / d}^{(1,1) *}-\frac{6}{5} \Omega_{s / d}^{(1,2) *}\right),
$$

where $m_{r}=m_{g} m_{p} /\left(m_{g}+m_{p}\right)$ is the reduced mass of a gas molecule and particle and $\Omega_{s / d}^{(1,1) *}$ and $\Omega_{s / d}^{(1,2) *}$ are the reduced collision integrals, which depend on the gas-particle interaction $[28,33]$. The subscripts " $s$ " and " $d$ " in Eq. (2) denote specular and diffuse scattering, respectively. Usually, the particle mass is much larger than that of a gas molecule $\left(m_{p} \gg m_{g}\right.$ ) and Eq. (2) reduces to Eq. (1) for both specular and diffuse scattering if rigid body collisions are assumed $\left(\Omega_{s / d}^{(1,1) *}=\Omega_{s / d}^{(1,2) *}=1[3,27]\right)$. Therefore, Eq. (2) is more general compared with Eq. (1) and can be applied in the free molecular regime in simple gases regardless of particle size.

In practical applications, many thermophoresis cases involve particles in gas mixtures, where the velocity distribution of the gas molecules depends on not only the temperature gradient but also on the concentration distribution [15]. Examples include the fabrication of thin films as sensing materials, where nanoparticles in gas mixtures are driven by temperature gradients and deposited on microelectronic chips [35]. In micro- and nanofabrications, thermophoresis can be used to protect the lithographic masks from nanoparticle contamination [36]. Other applications in materials synthesis and in the semiconductor area can be found in Ref. [37]. 
In the literature, little theoretical work has been reported on thermophoresis in gas mixtures due to the mathematical difficulty of solving the coupled Boltzmann equations for gas mixtures. In this work, we investigate the thermophoretic force on spherical particles in binary monatomic gas mixtures in the free molecular regime. Theoretical expressions for the thermophoretic force are derived on the basis of gas kinetic theory for specular and diffuse scattering. By setting the concentration of one component of the gas mixture to zero, it is shown that the analytical expressions are consistent with those for simple gases. The analytical formulas are valid for both micro- and nanoparticles.

\section{SECOND-ORDER APPROXIMATION OF BOLTZMANN EQUATION FOR BINARY GAS MIXTURES}

As will be shown later, the thermophoretic force can be obtained by calculating the momentum transfer during the collisions between the gas molecules and particle, which requires the velocity distribution of the gas molecules under a temperature gradient. For binary mixtures of monatomic gases, where the velocity distribution is not as simple as that of simple gases, the velocity distributions of the two components $f_{1}$ and $f_{2}$ satisfy the following Boltzmann equations [32,33]:

$$
\frac{\partial f_{i}}{\partial t}+\mathbf{c}_{i} \cdot \frac{\partial f_{i}}{\partial \mathbf{r}}+\mathbf{F}_{i} \cdot \frac{\partial f_{i}}{\partial \mathbf{c}_{i}}=\frac{\partial_{e} f_{i}}{\partial t}, \quad i=1,2,
$$

where $\mathbf{c}_{i}$ and $\mathbf{F}_{i}$ are the molecular velocity of and external force acting on species $i$, respectively, $\partial_{e} f_{i} / \partial t$ represents the change of $f_{i}$ due to molecular collisions and by which $f_{1}$ and $f_{2}$ are coupled to each other.

The first-order approximations of $f_{1}$ and $f_{2}$ are denoted by $f_{1}^{(0)}$ and $f_{2}^{(0)}$, respectively, which represent the equilibrium solutions and are given by the Boltzmann velocity distribution,

$$
f_{i}^{(0)}=n_{i}\left(\frac{m_{i}}{2 \pi k_{B} T}\right)^{3 / 2} \exp \left(-\frac{m_{i} v_{i}^{2}}{2 k_{B} T}\right), \quad i=1,2,
$$

where $n_{i}$ and $m_{i}$ are the number density and molecular mass of species $i$, respectively, and $v_{i}=\left|\mathbf{v}_{i}\right|$ represents the peculiar velocity of gas molecules, which is relative to the local mass velocity $\mathbf{c}_{0}$ of the gas mixture through $\mathbf{v}_{i}=\mathbf{c}_{i}-\mathbf{c}_{0}$ [32].

With the presence of a temperature gradient, the velocity distribution deviates from Eq. (4) and corresponds to the second-order approximation of $f_{i}, f_{i}^{(1)}$. According to the Chapman-Enskog theory [32], $f_{i}^{(1)}$ is given by

$$
f_{i}^{(1)}=f_{i}^{(0)} \Phi_{i}^{(1)}\left(\nabla T, \nabla n_{i}\right), \quad i=1,2,
$$

where $\Phi_{i}^{(1)}$ is a function of $\nabla T$ and $\nabla n_{i}$ if $\mathbf{c}_{0}$ is constant and given by

$$
\begin{aligned}
\Phi_{i}^{(1)}\left(\nabla T, \nabla n_{i}\right) & =-\mathbf{A}_{i} \cdot \nabla \ln T-\mathbf{D}_{i} \cdot \mathbf{d}_{i j}, \\
i, j & =1,2, \quad i \neq j,
\end{aligned}
$$

where the functions $\mathbf{A}_{i}$ and $\mathbf{D}_{i}$ are vectors and the vector $\mathbf{d}_{i j}$ are given by [32]

$$
\mathbf{d}_{i j}=\frac{n_{i}}{n} \nabla \ln p_{i}-\frac{\rho_{i} \rho_{j}}{p \rho}\left(\mathbf{F}_{i}-\mathbf{F}_{j}\right)-\frac{\rho_{i}}{p \rho} \nabla p,
$$

where $p=n k_{B} T$ and $p_{i}=n_{i} k_{B} T$ denote the total and partial pressures, respectively. Similarly, $\rho$ and $\rho_{i}=m_{i} n_{i}$ are the total and partial mass densities, respectively. If the external force is absent and the binary mixture is still, Eq. (7) reduces to

$$
\mathbf{d}_{i j}=\nabla x_{i}
$$

where, $x_{i}=n_{i} / n$ is the concentration. In steady state, $x_{i}$ is related to the temperature gradient through the thermal diffusion ratio $k_{T} \propto \mathscr{D}_{i}^{T} / \mathscr{D}_{i j}$, where $\mathscr{D}_{i}^{T}$ is the thermal diffusion coefficient of species $i$ and $\mathscr{D}_{i j}$ is the usual binary diffusion coefficient [33]

$$
\nabla x_{i}=-k_{T} \nabla \ln T .
$$

With Eqs. (8) and (9), $\Phi_{i}^{(1)}$ can be written as

$$
\Phi_{i}^{(1)}=-\tilde{\mathbf{A}}_{i} \cdot \nabla \ln T,
$$

where $\tilde{\mathbf{A}}_{i}=\mathbf{A}_{i}-k_{T} \mathbf{D}_{i}$, which can be expanded in a convergent Sonine polynomial [32,38,39]. If the first term in the polynomial is used, $\tilde{\mathbf{A}}_{i}$ can be expressed as

$$
\tilde{\mathbf{A}}_{i}=a_{i}\left(\frac{5}{2}-\zeta_{i}^{2}\right) \zeta_{i},
$$

where $\zeta_{i}=\mathbf{v}_{i} \sqrt{m_{i} /\left(2 k_{B} T\right)}$. In Eq. (11), $a_{i}$ depends on temperature $T$, mass $m_{i}$, and the intermolecular interaction potential (see Sec. III).

\section{THERMOPHORETIC FORCE IN BINARY GAS MIXTURES}

The thermophoretic force on a particle is related to momentum transfer between the gas molecules and particle. Consider a dilute binary gas mixture with a temperature gradient $\nabla T$. Assume that the mean velocity of the gas mixture is zero. When a particle of radius $R$ is introduced into the gas mixture with an instantaneous velocity $\mathbf{V}$, based on the theoretical framework given in Ref. [27], the thermophoretic force that is mainly due to species $i, \mathbf{F}_{T, i}\left(\mathbf{F}_{T, 1}\right.$ and $\mathbf{F}_{T, 2}$ are coupled, as discussed later), can be calculated as

$$
\mathbf{F}_{T, i}=m_{i p} \int_{\mathbf{v}_{i}} g_{i} \mathbf{g}_{i} f_{i}^{(0)} \Phi_{i}^{(1)} Q\left(g_{i}\right) d \mathbf{v}_{i},
$$

where $m_{i p}=m_{i} m_{p} /\left(m_{i}+m_{p}\right)$ is the reduced mass, $\mathbf{g}_{i}=\mathbf{v}_{i}-$ $\mathbf{V}$ is the relative velocity, and $Q\left(g_{i}\right)$ represents the collision cross section, which depends on the scattering scenario (i.e., specular or diffuse scattering) when gas molecules collide with the particle [27,32]. For specular scattering,

$$
Q_{s}\left(g_{i}\right)=2 \pi \int_{0}^{\infty}(1-\cos \chi) b d b,
$$

where $b$ is the impact parameter for the gas-particle collisions and $\chi$ is the angle of scattering given by [32]

$$
\chi=\pi-2 b \int_{r_{m}}^{\infty} \frac{d r}{r^{2} \sqrt{1-\frac{b^{2}}{r^{2}}-\frac{2 \varphi(r)}{m_{i p} g_{i}^{2}}}},
$$

where $r$ is the interaction distance, $r_{m}$ is the distance of the closest encounter, and $\varphi(r)$ is the interaction potential between 
the gas molecules and particle. For diffuse scattering, the collision cross section reads [27]

$$
\begin{aligned}
Q_{d}\left(g_{i}\right)= & 2 \pi\left[\int_{0}^{b_{0}}\left(1+\sqrt{\frac{\pi k_{B} T}{2 m_{i p} g_{i}^{2}}} \sin \frac{\chi}{2}\right) b d b\right. \\
& \left.+\int_{b_{0}}^{\infty}(1-\cos \chi) b d b\right],
\end{aligned}
$$

where $b_{0}$ is the critical impact factor and corresponds to the case of orbiting scattering $(\chi \rightarrow-\infty)$. For $b<b_{0}$, the gas molecules collide with the particle physically and diffuse scattering becomes possible. For $b>b_{0}$, grazing scattering takes place and the collision is considered to be specular [3,33].

\section{A. Analytical expressions for thermophoretic force}

By substituting Eqs. (4) and (10) into Eq. (12), the thermophoretic force $\mathbf{F}_{T, i}$ can be obtained as (a detailed derivation can be found in Appendix)

$$
\begin{gathered}
\mathbf{F}_{T, i}=-\frac{20 \sqrt{\pi} k_{B} R^{2} a_{i} n_{i}}{3}\left(\frac{m_{i}}{m_{i p}}\right)^{2}\left(\Omega_{i p}^{(1,1) *}-\frac{6}{5} \Omega_{i p}^{(1,2) *}\right) \nabla T, \\
i=1,2,
\end{gathered}
$$

where

$$
\Omega_{i p}^{(l, q) *}=\int_{0}^{\infty} \frac{e^{-\gamma_{i}^{2}} \gamma_{i}^{2 q+3} Q^{l}\left(g_{i}\right) d \gamma_{i}}{[(q+1) ! / 2]\left\{1-\left[1+(-1)^{l}\right] /(2+2 l)\right\} \pi R^{2}}
$$

is the reduced collision integral with $\gamma_{i}=g_{i} \sqrt{m_{i p} /\left(2 k_{B} T\right)}[27,32]$ and the subscript " $i p$ " in $\Omega_{i p}^{(l, q)}$ denotes that the interaction potential between species $i$ and the particle should be used in calculating the scattering angle $\chi$ in Eq. (14). The coefficient $a_{i}$ in Eq. (16) is given by [32]

$$
a_{i}=\frac{\alpha_{i} a_{j j}+\alpha_{j} a_{i j}}{\operatorname{det}|\mathscr{A}|}, \quad i, j=1,2, i \neq j,
$$

with $\alpha_{i}=-15 n_{i} \sqrt{2 k_{B} T / m_{i}} /\left(4 n^{2}\right)$ and matrix

$$
\mathscr{A}=\left[\begin{array}{ll}
a_{i i} & a_{i j} \\
a_{j i} & a_{j j}
\end{array}\right] .
$$

The elements of $\mathscr{A}$ are given by

$$
\begin{gathered}
a_{i i}=\frac{4 n_{i}^{2}}{n^{2}} \sqrt{\frac{k_{B} T}{\pi m_{i i}}} \Omega_{i i}^{(2,2)}+8 M_{j} \frac{n_{i} n_{j}}{n^{2}} \sqrt{\frac{k_{B} T}{2 \pi m_{i j}}} \Pi_{i i}, \\
a_{j j}=\frac{4 n_{j}^{2}}{n^{2}} \sqrt{\frac{k_{B} T}{\pi m_{j j}}} \Omega_{j j}^{(2,2)}+8 M_{i} \frac{n_{i} n_{j}}{n^{2}} \sqrt{\frac{k_{B} T}{2 \pi m_{i j}}} \Pi_{j j}, \\
a_{i j}=a_{j i}=-8\left(M_{i} M_{j}\right)^{-3 / 2} \frac{n_{i} n_{j}}{n^{2}} \sqrt{\frac{k_{B} T}{2 \pi m_{i j}}} \Pi_{i j},
\end{gathered}
$$

where $M_{i}=m_{i} /\left(m_{i}+m_{j}\right), m_{i j}=m_{i} m_{j} /\left(m_{i}+m_{j}\right)$, and $\Pi_{i i}, \Pi_{j j}$, and $\Pi_{i j}$ are the combinations of collision integrals:

$$
\begin{aligned}
\Pi_{i i}= & \frac{5}{4}\left(6 M_{i}^{2}+5 M_{j}^{2}\right) \Omega_{i j}^{(1,1)}-M_{j}^{2}\left(5 \Omega_{i j}^{(1,2)}-\Omega_{i j}^{(1,3)}\right) \\
& +2 M_{i} M_{j} \Omega_{i j}^{(2,2)}
\end{aligned}
$$

$$
\begin{aligned}
\Pi_{j j}= & \frac{5}{4}\left(6 M_{j}^{2}+5 M_{i}^{2}\right) \Omega_{i j}^{(1,1)}-M_{i}^{2}\left(5 \Omega_{i j}^{(1,2)}-\Omega_{i j}^{(1,3)}\right) \\
& +2 M_{i} M_{j} \Omega_{i j}^{(2,2)} \\
\Pi_{i j} & =\frac{55}{4} \Omega_{i j}^{(1,1)}-\left(5 \Omega_{i j}^{(1,2)}-\Omega_{i j}^{(1,3)}\right)-2 \Omega_{i j}^{(2,2)} .
\end{aligned}
$$

In Eqs. (18)-(23), $\Omega_{i j}^{(l, q)}$ is the collision integral for gas-gas interactions. For most gases, the Lennard-Jones (LJ) potential $\varphi_{i j}(r)=4 \varepsilon\left[(\sigma / r)^{12}-(\sigma / r)^{6}\right]$ can be used to describe the interactions, where $\varepsilon$ is the molecular binding energy and $\sigma$ is the collision diameter. The values of the collision integrals in Eqs. (18)-(23) based on the LJ potential can be found in standard handbooks about gas kinetic theory [33]. Therefore, the total thermophoretic force on the particle can be written as

$$
\begin{aligned}
\mathbf{F}_{T}= & -\frac{20 \sqrt{\pi}}{3} R^{2} k_{B} \nabla T\left[a_{1} n_{1}\left(\frac{m_{1}}{m_{1 p}}\right)^{2}\left(\Omega_{1 p}^{(1,1) *}-\frac{6}{5} \Omega_{1 p}^{(1,2) *}\right)\right. \\
& \left.+a_{2} n_{2}\left(\frac{m_{2}}{m_{2 p}}\right)^{2}\left(\Omega_{2 p}^{(1,1) *}-\frac{6}{5} \Omega_{2 p}^{(1,2) *}\right)\right] .
\end{aligned}
$$

It should be noted that $a_{1}$ and $a_{2}$ in Eq. (24) depend on the intermolecular interactions between the two gas species. Therefore, $\mathbf{F}_{T}$ in Eq. (24) is not a linear superposition of the thermophoretic forces caused by the gas components, as will be demonstrated later. For the sake of simplicity, we would like to rewrite Eq. (24) as

$$
\mathbf{F}_{T}=-\frac{20 \sqrt{\pi}}{3} R^{2} k_{B}\left(\Omega_{T, 1}^{*}+\Omega_{T, 2}^{*}\right) \nabla T,
$$

where $\Omega_{T, 1}^{*}$ and $\Omega_{T, 2}^{*}$ denote the first and second terms in the square bracket in Eq. (24). Note that the units of $\Omega_{T, 1}^{*}$ and $\Omega_{T, 2}^{*}$ are the same as $1 / R^{2}$, while the reduced collision integrals $\Omega_{i p}^{(l, q) *}$ in Eq. (24) are dimensionless.

It is noted that the thermophoretic force depends on how gas molecules are reflected upon collisions with the particle. Specular and diffuse scatterings are the two limiting cases. Usually diffuse scattering results in a larger force than specular scattering. For these two cases, the difference lies in the collision cross section [i.e., Eqs. (13) and (15)]. Therefore, the thermophoretic force for the two collision models can be expressed as

$$
\mathbf{F}_{T, s / d}=-\frac{20 \sqrt{\pi}}{3} R^{2} k_{B} \Omega_{T, s / d}^{*} \nabla T,
$$

where $\Omega_{T}^{*}=\Omega_{T, 1}^{*}+\Omega_{T, 2}^{*}$, which can be called the binary thermophoretic collision integral and the subscript " $s / d$ " denotes that the specular or diffuse collision cross section, Eqs. (13) or (15), should be used in calculating the collision integrals in Eq. (24).

If the concentration of one component of the gas mixture approaches zero, it is expected that Eq. (26) converges to Eq. (2) for simple gases. In Eq. (26), if, for example, $x_{2} \rightarrow 0$ and $x_{1} \rightarrow 1$, it can be shown that $a_{1} n_{1}=-15 \sqrt{2 \pi} /\left(16 \Omega_{11}^{(2,2)}\right)$ and $\mathbf{F}_{T, s / d}$ simplified to

$$
\mathbf{F}_{T, s / d}=\frac{25 \sqrt{2} \pi}{4 \Omega_{11}^{(2,2)}} R^{2} k_{B} \nabla T\left(\Omega_{1 p}^{(1,1) *}-\frac{6}{5} \Omega_{1 p}^{(1,2) *}\right)_{s / d},
$$

by assuming that $m_{p} \gg m_{1}$. Equation (27) becomes the same as Eq. (2) given that the conductivity of the gas 
$\kappa=75 k_{B}^{2} T /\left(32 \sqrt{k_{B} T m_{1} / \pi} \Omega_{11}^{(2,2)}\right)$ [32]. It is worth mentioning that Eq. (26) is based on the second-order approximation of the velocity distribution function. For extremely high-temperature gradients, the higher-order terms in the velocity distribution may need to be considered. However, in most cases, the error caused by the higher-order terms is below $10 \%$ [27], which is expected to be minor compared with the uncertainty involved in the gas-particle interaction potential.

\section{B. Thermophoretic force on microparticles with rigid body collisions}

Equation (24) represents a very general expression for the thermophoretic force, where the intermolecular interactions are considered. For microspheres, the gas-particle interaction is unimportant $[3,26,27]$ and the analytical results of the collision integrals in Eq. (24) can be obtained as

$$
\Omega_{1 p}^{(1,1) *}=\Omega_{1 p}^{(1,2) *}=\Omega_{2 p}^{(1,1) *}=\Omega_{2 p}^{(1,2) *}=1 .
$$

Hence, the thermophoretic force on large spheres with rigid body collisions in binary gas mixtures can be written as

$$
\mathbf{F}_{T}=\frac{4 \sqrt{\pi}}{3} R^{2} k_{B} \nabla T\left(a_{1} n_{1}+a_{2} n_{2}\right),
$$

provided that the mass of the particle is much larger than that of the gas molecules. It is easy to check that Eq. (29) is consistent with the Waldmann equation (1), for simple gases as $n_{1}$ or $n_{2}$ goes to zero.

\section{Thermophoretic force on nanoparticles}

For nanoparticles, the gas-particle interactions may become significant and the collision integrals $\Omega_{i p}^{(l, q) *}$ in Eq. (24), which depend on the interaction potentials, should be evaluated to calculate the thermophoretic force. The LJ potential may be too simple to model the gas-particle interaction, for which the Rudyak-Krasnolutski 9-3 potential probably is a good choice, although it has limitations. Detailed information about the model can be found in Refs. [26,27,40].

Herein, as an example, we present the thermophoretic force on a silver nanoparticle in dilute $\mathrm{He}$-Ar gas mixtures. The binding energy $\varepsilon$ and collision diameter $\sigma$ for $\mathrm{He}$ and Ar are set to $\varepsilon_{1} / k_{B}=10.0 \mathrm{~K}, \sigma_{1}=2.55 \AA$ and $\varepsilon_{2} / k_{B}=114.0 \mathrm{~K}$, $\sigma_{2}=3.47 \AA$, respectively [41,42]. For He-Ar interaction, the Lorentz-Berthelot mixing rules $\sigma_{12}=\left(\sigma_{1}+\sigma_{2}\right) / 2$ and $\varepsilon_{12}=$ $\sqrt{\varepsilon_{1} \varepsilon_{2}}$ are employed. To evaluate the gas-particle collision integrals $\Omega_{i p}^{(1,1) *}$ and $\Omega_{i p}^{(1,2) *}, \sigma_{p}=2.574 \AA$ and $\varepsilon_{p} / k_{B}=$ $4075.0 \mathrm{~K}$ are used for $\mathrm{Ag}$ nanoparticles, and $\sigma_{i p}=\left(\sigma_{i}+\sigma_{p}\right) / 2$ and $\varepsilon_{i p}=\sqrt{\varepsilon_{i} \varepsilon_{p}}[26,27,43]$. The radius of the $\mathrm{Ag}$ nanoparticle is set as $R=10 \sigma_{1 p}$.

Figure 1 shows the quantity $\Omega_{T}^{*} R^{2}$, which is dimensionless, as a function of the mean temperature of the gas mixture for specular [Fig. 1(a)] and diffuse [Fig. 1(b)] scatterings at different concentrations of $\mathrm{He} x_{1}$. Figure 1 indicates that the thermophoretic force for diffuse scattering is larger than that for specular scattering, given the temperature gradient and mean temperature. This is the same as the case in simple gases. It is also reasonable that $\mathbf{F}_{T}$ increases with increasing mean temperature because the momentum transfer is enhanced at high temperatures. Furthermore, it is seen that
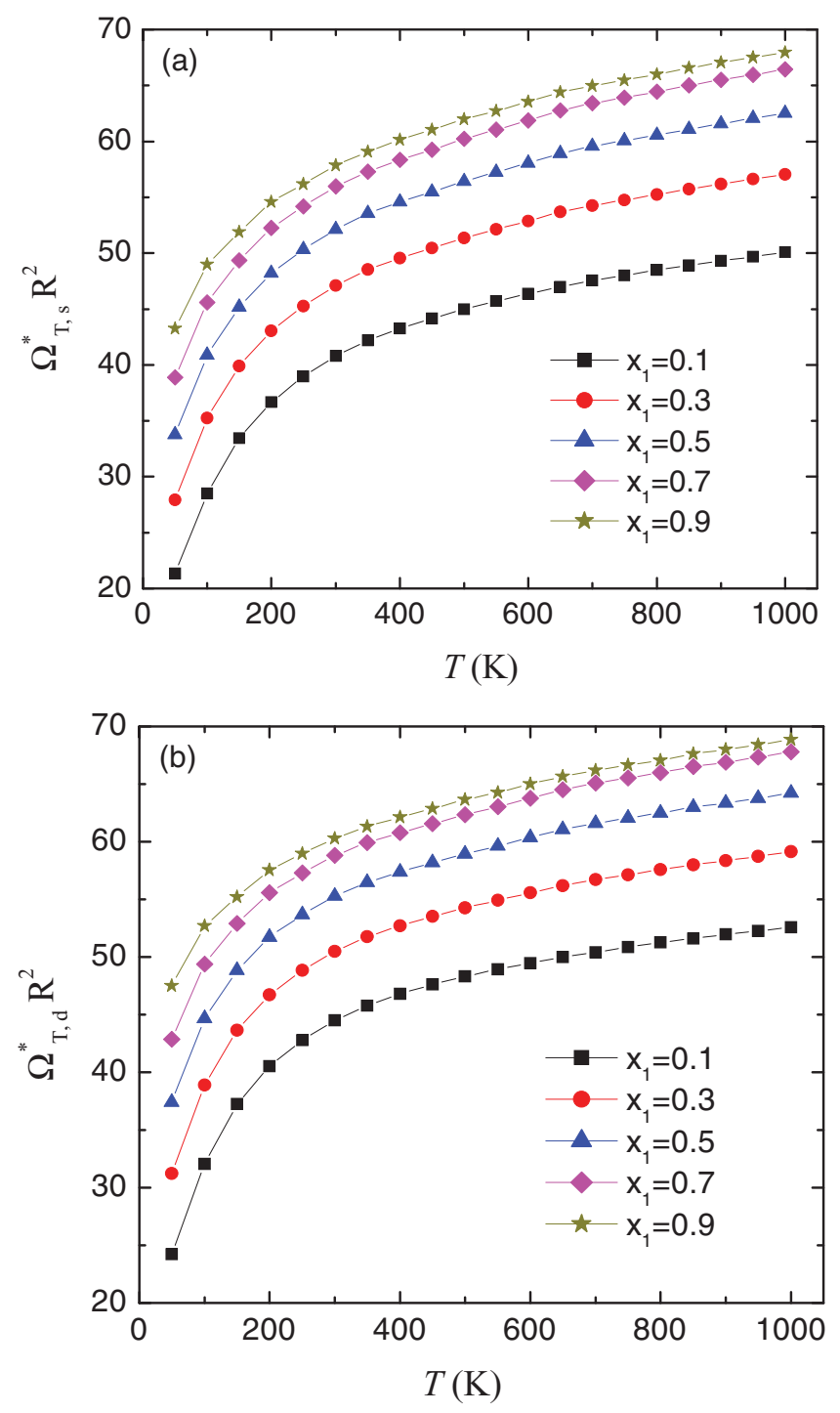

FIG. 1. (Color online) $\Omega_{T}^{*} R^{2}$ as a function of the mean temperature of the gas mixture $T$ at different He concentrations $x_{1}$ for specular (a) and diffuse (b) scattering.

the thermophoretic force on the Ag nanoparticle depends on the concentrations of the mixture. For the case in Fig. 1, it increases with increasing He concentration $x_{1}$ for a given mean temperature. This, we believe, is the consequence of gas-gas and gas-particle interactions, which are very complicated. Due to the complexity of the collision integrals, it is difficult to offer a criterion about how the thermophoretic force qualitatively depends on the concentration of the mixture. Further investigations on this point are required.

In Fig. 2, $\Omega_{T}^{*} R^{2}$ and $\Omega_{T, i}^{*} R^{2}$ are depicted as a function of He concentration $x_{1}$ with mean temperature $T=300 \mathrm{~K}$. It is seen that $\mathbf{F}_{T, 1}(\mathrm{He})$ increases while $\mathbf{F}_{T, 2}(\mathrm{Ar})$ decreases nonlinearly as $x_{1}$ increases and that $\mathbf{F}_{T, 1}$ is more sensitive to $x_{1}$ compared with $\mathbf{F}_{T, 2}$. This guarantees that the total thermophoretic force $\mathbf{F}_{T}$ increases with increasing $x_{1}$, as shown in Fig. 1. The dependence of $\Omega_{T}^{*} R^{2}$ and $\Omega_{T, i}^{*} R^{2}$ on $x_{1}$ follows the same fashion for specular [Fig. 2(a)] and diffuse [Fig. 2(b)] scatterings. Due to the nonlinear dependence, there 

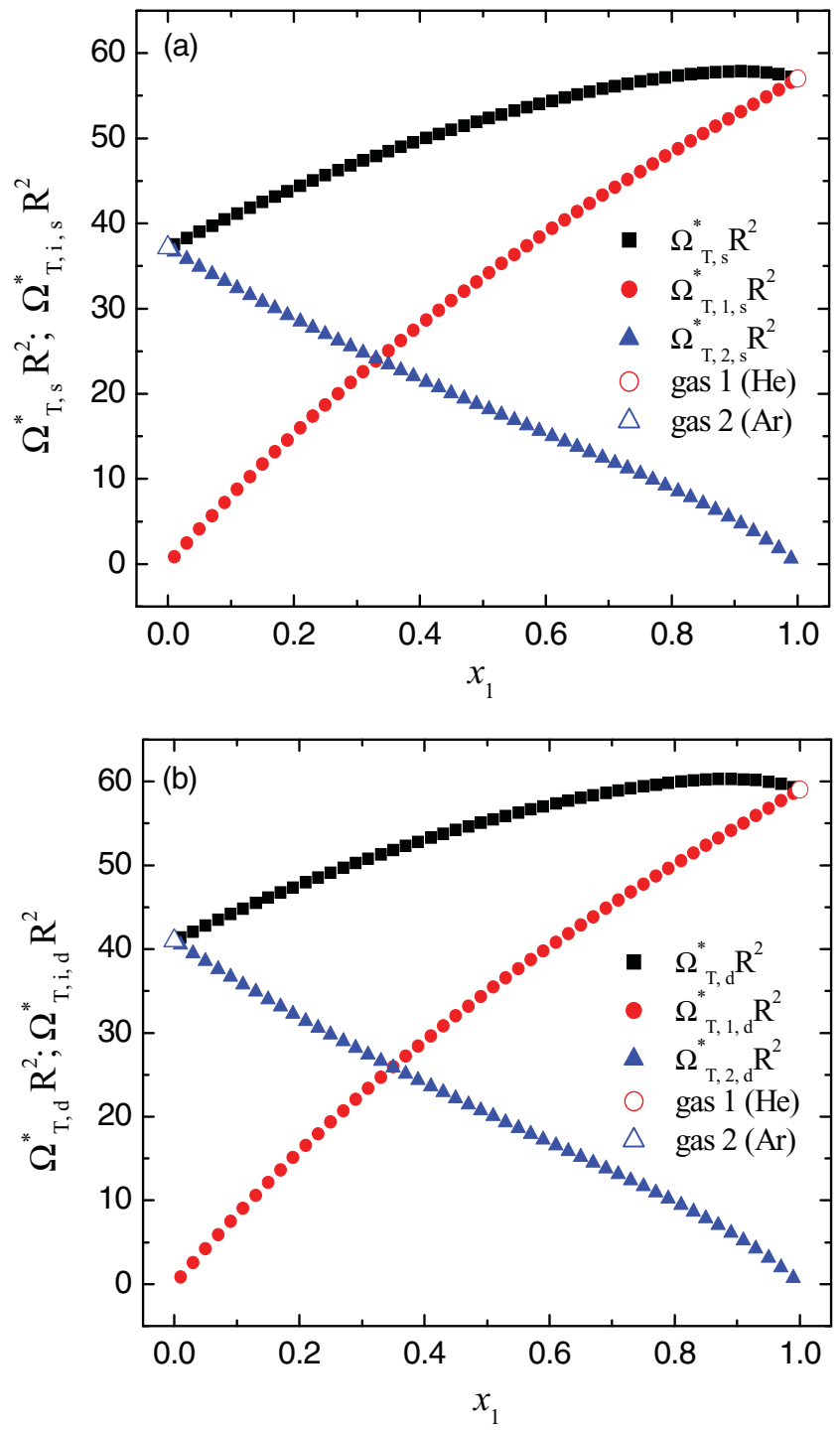

FIG. 2. (Color online) Dependence of $\Omega_{T}^{*} R^{2}$ and $\Omega_{T, i}^{*} R^{2}$ on $\mathrm{He}$ concentrations $x_{1}$ for specular (a) and diffuse (b) scattering at $T=$ $300 \mathrm{~K}$. Open symbols represent the results in pure simple gases.

is a maximum at $x_{1} \sim 0.9$, where $\mathbf{F}_{T}$ is even larger than its counterparts in pure $\mathrm{He}$ and Ar, which are illustrated by open symbols in Fig. 2. Figure 2 also shows that Eq. (26) converges to Eq. (2) as $x_{1}$ converges to 0 or 1 .

Finally, we would like to point out that the thermophoretic force given by Eq. (24) could be negative $\left(\Omega_{i p}^{(1,1) *}>\right.$

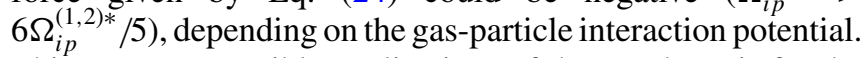
This suggests possible applications of thermophoresis for the separation of particles of different materials. The negative thermophoretic force has been theoretically proven possible for small Knudsen numbers based on the Boltzmann-KrookWelander model of the Boltzmann equation, although it is too weak to be observed experimentally [4,5,44-47]. It has also been argued that the negative thermophoretic force might be an artifact due to theoretical approximations [48]. Nonetheless, our current analyses indicate that the thermophoretic force could be negative in the free molecular regime if appropriate gas-particle interaction potentials are employed. The negative thermophoretic force is a nontrivial issue and requires intensive investigations.

\section{CONCLUSIONS}

In summary, we have theoretically derived the thermophoretic force on spherical particles in binary mixtures of monatomic gases in the free molecular regime by using gas kinetic theory. The formulas converge to those in simple gases and can be reduced to the cases of rigid body collision. The analytical expressions are valid for particles of size ranging from the nano- to microscale provided that the case is in the free molecular regime $(\mathrm{Kn} \gg 1)$.

\section{ACKNOWLEDGMENTS}

This work was supported by the Hong Kong Innovation and Technology Fund under Grant No. ITS/529/09 and the postdoctoral fellowship matching fund of HKUST.

\section{APPENDIX: DERIVATION OF THERMOPHORETIC FORCE}

Substituting Eqs. (4) and (10) into Eq. (12), the thermophoretic force $\mathbf{F}_{T, i}$ reads

$\mathbf{F}_{T, i}=m_{i p} \int_{\mathbf{v}_{i}} g_{i} \mathbf{g}_{i} f_{i}^{(0)} Q\left(g_{i}\right) a_{i}\left(\zeta_{i}^{2}-\frac{5}{2}\right) \zeta_{i} \cdot \nabla \ln T d \mathbf{v}_{i}$.

It is reasonable to assume that the variation in the drift velocity $V$ is much smaller than the peculiar velocity of the gas molecules $v_{i}\left(V \ll v_{i}\right)$. If $\mathbf{V}$ is in the same direction as the temperature gradient and $\phi$ and $\theta$ are the colatitude and azimuthal angles of $\mathbf{g}_{i}$, respectively, in a reference frame in which $\boldsymbol{V}$ is in the $z$ direction [24,26], it can be shown that

$$
d \mathbf{v}_{i} \approx d \mathbf{g}_{i}=g_{i}^{2} \sin \phi d \phi d \theta d g_{i}
$$

Since the direction of the thermophoretic force is collinear with that of the temperature gradient, only the component in the $\mathbf{V}$ direction of $\mathbf{g}$ needs to be considered. This simplifies Eq. (A1) to

$$
\begin{aligned}
\mathbf{F}_{T, i}= & m_{i p} \int_{\mathbf{g}_{i}} g_{i}^{4} f_{i}^{(0)} Q\left(g_{i}\right) a_{i}\left(\zeta_{i}^{2}-\frac{5}{2}\right) \zeta_{i} \\
& \cdot \mathbf{u} \nabla \ln T \sin \phi \cos \phi d \phi d \theta d g_{i},
\end{aligned}
$$

where $\mathbf{u}$ is the unit vector in the $\mathbf{V}$ direction. In consideration of $V \ll v_{i}$, we have $v_{i}^{2}=g_{i}^{2}+V^{2}+2 g_{i} V \cos \phi \approx$ $g_{i}^{2}+2 g_{i} V \cos \phi$, and the exponential term in $f_{i}^{(0)}$ and the term $\left(\zeta_{i}^{2}-\frac{5}{2}\right) \zeta_{i} \cdot \mathbf{u}$ can be simplified as [26]

$$
\begin{aligned}
\exp \left(-\zeta_{i}^{2}\right) & \approx \exp \left(-\gamma_{i}^{2}\right) \text { and }\left(\zeta_{i}^{2}-\frac{5}{2}\right) \zeta_{i} \cdot \mathbf{u} \\
& \approx \sqrt{\frac{m_{i}}{2 k_{B} T}}\left(\gamma_{i}^{2}-\frac{5}{2}\right) g_{i} \cos \phi
\end{aligned}
$$


By substituting Eq. (A4) into Eq. (A3), we obtain

$$
\begin{aligned}
\mathbf{F}_{T, i}= & m_{i p} \int_{\mathbf{g}_{i}} g_{i}^{4} f_{i}^{(0)} Q\left(g_{i}\right) a_{i}\left(\zeta_{i}^{2}-\frac{5}{2}\right) \zeta_{i} \\
& \cdot \mathbf{u} \nabla \ln T \sin \phi \cos \phi d \phi d \theta d g_{i} \\
= & \frac{4 k_{B} T a_{i} n_{i}}{\sqrt{\pi}}\left(\frac{m_{i}}{m_{i p}}\right)^{2} \nabla \ln T \int_{0}^{\infty} \gamma_{i}^{5} e^{-\gamma_{i}^{2}}\left(\gamma_{i}^{2}-\frac{5}{2}\right) \\
& \times Q\left(g_{i}\right) d \gamma_{i} \int_{0}^{\pi} \sin \phi \cos ^{2} \phi d \phi
\end{aligned}
$$

$$
\begin{aligned}
= & -\frac{20 k_{B} a_{i} n_{i}}{3 \sqrt{\pi}}\left(\frac{m_{i}}{m_{i p}}\right)^{2}\left\{\int_{0}^{\infty} \gamma_{i}^{5} e^{-\gamma_{i}^{2}} Q\left(g_{i}\right) d \gamma_{i}\right. \\
& \left.-\frac{2}{5} \int_{0}^{\infty} \gamma_{i}^{7} e^{-\gamma_{i}^{2}} Q\left(g_{i}\right) d \gamma_{i}\right\} \nabla T \\
= & -\frac{20 k_{B} a_{i} n_{i}}{3 \sqrt{\pi}}\left(\frac{m_{i}}{m_{i p}}\right)^{2}\left\{\Omega_{i p}^{(1,1)}-\frac{2}{5} \Omega_{i p}^{(1,2)}\right\} \nabla T \\
= & -\frac{20 \sqrt{\pi} k_{B} R^{2} a_{i} n_{i}}{3}\left(\frac{m_{i}}{m_{i p}}\right)^{2}\left(\Omega_{i p}^{(1,1) *}-\frac{6}{5} \Omega_{i p}^{(1,2) *}\right) \nabla T,
\end{aligned}
$$

which is Eq. (16).
[1] C. Ludwig, Sitzber. Kaiserl. Akad. Wiss. Math.-Natwiss. K1. 20, 539 (1856).

[2] J. Tyndall, Proc. R. Inst. 6, 3 (1870).

[3] Z. Li and H. Wang, Phys. Rev. E 68, 061206 (2003).

[4] E. Bringuier and A. Bourdon, Physica A 385, 9 (2007).

[5] F. Zheng, Adv. Colloid Interface Sci. 97, 255 (2002).

[6] P. S. Epstein, Z. Phys. 54, 537 (1929).

[7] P. S. Epstein, Phys. Rev. 23, 710 (1924).

[8] R. A. Millikan, Phys. Rev. 22, 1 (1924).

[9] J. R. Brock, J. Colloid Sci. 17, 768 (1962).

[10] L. Waldmann, Z. Naturforsch. A 14a, 589 (1959).

[11] E. A. Mason and S. Chapman, J. Chem. Phys. 36, 627 (1962).

[12] L. Monchick, K. S. Yun, and E. A. Mason, J. Chem. Phys. 39, 654 (1963).

[13] C. N. Davies, Aerosol Science (Academic, New York, 1966).

[14] B. K. Annis and E. A. Mason, J. Aerosol Sci. 6, 105 (1975).

[15] L. A. Viehland and E. A. Mason, J. Aerosol Sci. 8, 381 (1977).

[16] N. T. Tong, J. Colloid Interface Sci. 51, 143 (1975).

[17] W. Li and E. J. Davis, J. Aerosol Sci. 26, 1063 (1995).

[18] W. Li and E. J. Davis, J. Aerosol Sci. 26, 1085 (1995).

[19] S. K. Loyalka, J. Aerosol Sci. 23, 291 (1992).

[20] K. Yamamato and Y. Ishihara, Phys. Fluids 31, 3618 (1988).

[21] M. Zurita-Gotor, J. Aerosol Sci. 37, 283 (2006).

[22] D. Braun and A. Libchaber, Phys. Rev. Lett. 89, 188103 (2002).

[23] S. Duhr and D. Braun, Proc. Natl. Acad. Sci. USA 103, 19678 (2006).

[24] S. Duhr and D. Braun, Phys. Rev. Lett. 96, 168301 (2006).

[25] M. Braibanti, D. Vigolo, and R. Piazza, Phys. Rev. Lett. 100, 108303 (2008).

[26] Z. Li and H. Wang, Phys. Rev. E 68, 061207 (2003).

[27] Z. Li and H. Wang, Phys. Rev. E 70, 021205 (2004).
[28] Z. Li and H. Wang, Phys. Rev. Lett. 95, 014502 (2005).

[29] H. Wang, Ann. New York Acad. Sci. 1161, 484 (2009).

[30] H. Brenner, Phys. Rev. E 82, 036325 (2010).

[31] H. Brenner, Phys. Rev. E 72, 061201 (2005).

[32] S. Chapman and T. G. Cowling, The Mathematical Theory of Non-Uniform Gases (Cambridge University Press, Cambridge, 1970).

[33] J. O. Hirschfelder, C. F. Curtiss, and R. B. Bird, Molecular Theory of Gases and Liquids (Wiley, New York, 1954).

[34] G. A. Bird, Molecular Gas Dynamics (Oxford University Press, London, 1976).

[35] L. Mädler, H. K. Kammler, R. Mueller, and S. E. Pratsinis, J. Aerosol Sci. 33, 369 (2002).

[36] D. E. Dedrick, E. W. Beyer, D. J. Rader, L. E. Klebanoff, and A. H. Leung, J. Vac. Sci. Technol. B 23, 307 (2005).

[37] L. Mädler and S. K. Friedlander, Aerosol Air Qual. Res. 7, 304 (2007).

[38] D. Burnett, Proc. Lond. Math. Soc. 39, 385 (1935).

[39] D. Burnett, Proc. Lond. Math. Soc. 40, 382 (1936).

[40] V. Ya. Rudyak and S. L. Krasnolutski, Dokl. Phys. 46, 897 (2001).

[41] Z. Li, Phys. Rev. E 79, 026312 (2009).

[42] H. Hippler, J. Troe, and H. J. Wendelken, J. Chem. Phys. 78, 6709 (1983).

[43] P. M. Agrawal, B. M. Rice, and D. L. Thompson, Surf. Sci. 515, 21 (2002).

[44] H. A. Dwyer, Phys. Fluids 10, 977 (1967).

[45] Y. Sone, Phys. Fluids 15, 1418 (1972).

[46] Y. Sone and K. Aoki, Progress in Astronautics and Aeronautics, Rarefied Gas Dynamics, Vol. 74 (New York, 1981), p. 489.

[47] D. Lhuillier, Physica A 390, 1221 (2011).

[48] S. K. Loyalka, J. Aerosol Sci. 23, 291 (1992). 